$0022-3018 / 88 / 1769-0558 \$ 02.00 / 0$

The Journat of Nervotes ant Mental Disease

Copyright $\bigcirc 1988$ by The Williams \& Wilkins $C 0$.

\title{
High Potency Neuroleptics and Violence in Schizophrenics
}

\author{
John N. HerRera, Ph.D.', John J. SRAMEK, Pharm.D. ${ }^{2}$, Jerome F. COSTA, M.D, SWati Roy, Ph.D. ${ }^{3}$, \\ CHRIS W. HEH, M.D. ${ }^{4}$, AND BICH N. NGUYEN, R.N. ${ }^{3}$
}

\begin{abstract}
In a controlled study, inpatient violence was measured during placebo, high-potency (haloperidol) and low-potency (chlorpromazine or clozapine) neuroleptics. Some patients had a marked increase in violent behavior with the moderately high-dose haloperidol, but not with low-potency neuroleptics. The authors discuss reasons for the increased violence with haloperidol, including akathisia and drug-induced behavioral toxicity.
\end{abstract}

Until recently, it was widely believed that the mentally ill were either less violent or no more violent than the general population. Recent findings, however, suggest that violence is quite common in psychiatric hospitals (Yesavage, 1984), and considerable emphasis is now being placed on the evaluation (Yesavage, 1983), treatment (Felthous, 1984), and prediction of violence (Mulvey and Lidz, 1984) and on the resultant legal and ethical issues. Although the potential drug treatments for violent behavior are many, neuroleptic drugs are often considered the treatment of choice when violence stems from the psychotic condition (Dubin, 1981; Gunn, 1979). However, there are few guidelines by which the clinician can choose an optimal neuroleptic. According to anecdotal reports, some clinicians prefer thioridazine (Itil and Wadad, 1975), haloperidol (King, 1981), or clopenthixol (Yar-Khan, 1981); others prefer highpotency antipsychotics to low-potency antipsychotics because of a wider margin of safety when used in high dosage (Csernansky et al., 1985). In one study of 33 aggressive mentally retarded people, an unmarketed benzazepine derivative was noted to cause less violent behavior than treatment with thioridazine (Elie et al., 1980). However, we are unaware of any similar comparisons of controlled studies with neuroleptics in schizophrenic patients. We therefore undertook systematic measurement of violence in patients who were to receive both high and low-potency neuroleptics in a controlled study assessing the effectiveness of clozapine and chlorpromazine in schizophrenic patients who were prospectively determined to be treatment resistant by failure to respond to moderately high-dose haloperidol.

\section{Inclusion}

\section{Methods}

Included in the study were 16 male patients who

${ }^{1}$ Clinical Research Unit, Metropolitan State Hospital, 11400 Norwalk Boulevard, Norwalk, California 90250 . Send reprint requests to Dr. Herrera.

${ }^{2}$ Department of Drug Research, Metropolitan State Hospital.

${ }^{3}$ Clinical Research Unit, Metropolitan State Hospital.

4 Department of Psychiatry, University of California, Irvine. met DSM-III criteria for schizophrenia and were proven resistant to previous neuroleptic treatment efforts. The mean age of the patients was 33.3 years (range, 25 to 44 years) the mean age at the first hospitalization was 18.8 years (range, 12 to 27 years), and the mean number of years since the first onset of psychosis was 15.4 (range, 7 to 24). The mean length of the current hospitalization was 83.9 weeks (range, 40 to 445 ), and the mean number of prior psychiatric hospitalizations was 10.5 (range, 3 to 25 ). A retrospective chart review revealed that these patients did not have unusual histories of violence.

\section{Treatment Resistance}

Treatment resistance was determined on the basis of documented failure to respond adequately to treatment with at least three drugs (from two different neuroleptic classes) for durations of 6 weeks or longer at minimal daily doses equivalent to approximately $1000 \mathrm{mg} /$ day of chlorpromazine during the preceding 5 years, with no period of good response to antipsychotic therapy during this time. At least two of these three drug experiences would have to have occurred in the preceding 2.5 years. Documentation of this information was done by systematic extraction of medical records for the preceding 5 years by experienced research assistants.

\section{Exclusion}

Excluded from the study were patients who did not exhibit active symptoms, or patients with organic cerebral brain disease or mental retardation by DSMIII criteria. Also, patients younger than 18, older than 55 , those actively abusing illicit drugs or alcohol in the 2 weeks prior to entrance in the study, or those physically ill were excluded.

\section{Procedure}

At the time of the study, all patients were part of a larger multicenter clozapine investigation that included a placebo washout period, a high-potency med- 
ication period (haloperidol), and a low-potency medication period (clozapine or chlorpromazine). Identified study candidates who had given informed consent were administratively transferred to our research unit and then entered a 14-day washout period receiving only placebo capsules (t.i.d.).

\section{Study Periods}

The placebo washout was followed by a 6 -week clinical trial of fixed-dose haloperidol. For the first 5 days, dosage was increased by $5 \mathrm{mg} /$ day up to $20 \mathrm{mg} /$ day and then stablized for 3 days. Dosages were then increased by $10 \mathrm{mg} /$ day every three days to a maximum of $60 \mathrm{mg} / \mathrm{day}$, where they remained for the rest of the 6-week period. This study was followed by a second placebo period and entry into a 6 -week double-blind medication trial. Following a week's titration period, patients received fixed dosages of either clozapine $(900$ $\mathrm{mg} /$ day) or chlorpromazine (1800 mg/day). Patients given haloperidol and chlorpromazine also received benztropine $(6 \mathrm{mg} / \mathrm{day})$ while on these drugs.

\section{Clinical Change}

Clinical change was assessed on the following scales, completed by research staff at entrance and weekly during the placebo washout period: Brief Psychiatric Rating Scale (BPRS; Overall and Gorham, 1962), Simpson-Angus Rating Scale for Extrapyramidal Effects (1970), and the Abnormal Involuntary Movement Scale (AIMS; Guy, 1976). Senior nursing staff completed the Lion's Scale of Inpatient Violence (Lion et al., 1981), on a daily basis. The Lion's Scale is based on daily observations of a patient's behavior and a review of medical records. Episodes of hostile and assaultive behavior are extracted and quantified into the following subscales: physical (assaults against people, property, or self), verbal (assaults against patients or staff), and total assaults (verbal and physical assaults); the scale is then saved on a weekly basis.

\section{Results}

One-way repeated analyses of variance were performed on the Lion's sum weekly scores for the three study periods (placebo, haloperidol, and low potency medication trials) and the results revealed a borderline significant effect $(F=2.81, p<.076)$. For the purpose of subsequent analysis of the placebo and two drug trial differences, $t$-tests were chosen. These comparisons yielded significant differences between the haloperidol medication and placebo period $\bar{X} \pm$ SD: haloperidol, $5.25 \pm 8.3$; placebo, $1.94 \pm 2.5 ; t=3.312, p$ $<.052$ ) and the haloperidol and low potency medication periods (low-potency, $1.56 \pm 2.1 ; t=3.68, p<.034$ ). No difference was found between the low-potency medication and placebo period $(t=.488)$. Examination of the direction of the means for the three study periods indicated that significantly more violent episodes had occurred during the haloperidol period than during the placebo or low-potency period. Inspection of the Lion's data for the haloperidol period revealed the presence of two distinct patient groups using a Lion's score of 3 or above for the violent group: a violent ( $N$ $=7)$ and nonviolent schizophrenic group $(N=9)$. Comparisons ( $t$-test; Table 1 ) yielded significant differences between the two patient groups on Lion's total $(p<.004)$, physical $(p<.004)$, and verbal $(p<$ $.007)$ subscales. No differences were found between these patients on the Lion's subscales during the placebo period.

The results of the BPRS are found in Table 2, which provides means and standard deviations for the violent and nonviolent schizophrenic patients at the end of the 6 -week haloperidol medication trial. Significant differences were revealed on the BPRS total $(p<.18)$ and paranoia $(p<.03)$ subscales; and borderline significance was reached on the anxiety subscale $(p<.12)$. Examination of the direction of the BPRS total and subscale means (Table 2) indicates that the violent schizophrenic patients presented more pathological clinical pictures with prominent paranoid features and reported more subjective stress. No differences were found between these patients on the BPRS subscales during the placebo period. The Neurological Rating Scale and the scale item "akathisia" were also examined at the end of each period. The results for the haloperidol period suggested fewer extrapyramidal symptoms ( $\bar{X} \pm \mathrm{SD}$ ): nonviolent, $7.55 \pm 5.87$; violent,

TABLE 1

Lion's Scores for Violent and Nonviolent Schizophrenic Patients in High-Potency Medication Period ${ }^{a}$

\begin{tabular}{lrrc}
\hline Lion's Subscale & Violent Patients & Nonviolent Patients & $t$ \\
\hline Verbal assaults & $6.14 \pm 5.64$ & $1.33 \pm .71$ & $3.09^{* * *}$ \\
Physical assaults & $5.28 \pm 4.61$ & $.11 \pm .33$ & $3.39^{* * *}$ \\
Total assaults & $11.42 \pm 9.67$ & $.44 \pm .88$ & $3.42^{* * *}$ \\
\hline
\end{tabular}

${ }^{"} \bar{X} \pm \mathrm{SD}$.

$* * * p<.01$.

TABLE 2

BPRS Scores for Violent and Nonviolent Schizophrenic Patients in High-Potency Medication Period

\begin{tabular}{lcccc}
\hline Anxiety & 2.93 & \pm .79 & $2.22 \pm .94$ & $1.59^{*}$ \\
Depression & 2.73 & \pm 1.51 & $2.33 \pm .71$ & .71 \\
Paranoia & 4.14 & \pm .1 .34 & $2.74 \pm 1.01$ & $2.39^{* *}$ \\
Agitation & $2.14 \pm 1.86$ & $1.22 \pm .44$ & 1.44 \\
Psychosis & $4.65 \pm .85$ & $4.11 \pm .65$ & 1.45 \\
Withdrawal & $3.78 \pm 1.15$ & $3.09 \pm .72$ & 1.48 \\
Total & $67.00 \pm 13.19$ & $53.66 \pm 6.65$ & $2.65^{* *}$ \\
\hline
\end{tabular}

${ }^{a} \bar{X} \pm \mathrm{SD}$.

$* p<.1 ; * * p<.05$. 
$12.92 \pm 5.13$ ) and less akathisia (nonviolent $.889 \pm$ 1.69; violent, $2.71 \pm 3.98$ ) in the nonviolent group during the haloperidol period, but there were no overall statistically significant differences between the groups.

The results of the BPRS at the end of the 6-week clinical trial of low-potency medication are found in Table 3, which provides means and standard deviations for the violent and nonviolent schizophrenic patients. Matched $t$-test comparisons were used to examine the impact of low-potency medication on the violent and nonviolent subjects. In comparison with the haloperidol period, violent patients significantly improved on the BPRS total $(p<.05)$ and paranoia $(p$ $<$.04) subscale, and borderline significance was reached on the thought disorder $(p<.06)$ subscales. Examination of the nonviolent patients' haloperidol $v s$. lowpotency periods failed to reveal significant differences on any of the BPRS subscales. Included in the lowpotency group were three violent and four nonviolent clozapine patients and four violent and five nonviolent chlorpromazine patients.

\section{Discussion}

In this study of chronic schizophrenics, inpatient violence was measured during placebo, haloperidol, and low-potency neuroleptic drug periods. Analysis of the results revealed that patients were significantly more violent during haloperidol treatment than during the other two periods. Inspection of the Lion's data for the haloperidol period indicated the presence of two distinct patient groups: violent and nonviolent. The violent subgroup also deteriorated on their BPRS scores during the haloperidol period but not during the placebo or low-potency periods. Therefore, it appears that some patients have an increase in violent behavior when given moderately high-dose haloperidol.

There are several possible explanations for these results. Obviously, if patients worsened psychiatrically during any treatment period, other concomitant or linked behaviors, such as violence, might also be

TABLE 3

BPRS Scores for Violent and Nonviolent Schizophrenic Patients in High-and Low-Potency Medication Period

\begin{tabular}{lccccccc}
\hline & \multicolumn{3}{c}{ Violent } & & \multicolumn{3}{c}{ Nonviolent } \\
\cline { 2 - 4 } \cline { 6 - 7 } BPRS Subscale & High & Low & $t$ & & High & Low & $t$ \\
\hline Anxiety & 2.93 & 2.35 & 2.33 & & 2.22 & 2.33 & .34 \\
Depression & 2.73 & 2.92 & .36 & & 2.33 & 2.13 & .65 \\
Paranoia & 4.14 & 2.84 & $2.52^{* *}$ & & 2.74 & 2.46 & .64 \\
Agitation & 2.14 & 1.43 & 1.69 & & 1.22 & 1.55 & 1.00 \\
Psychosis & 4.65 & 3.94 & $2.19^{*}$ & & 4.11 & 3.64 & 1.28 \\
Withdrawal & 3.78 & 3.27 & 1.23 & & 3.09 & 3.14 & .12 \\
Total & 67.00 & 55.57 & $2.33^{* *}$ & 53.66 & 51.33 & .668 \\
\hline
\end{tabular}

${ }^{*} p<.1 ;{ }^{* *} p<.05$ expected to worsen. However, these patients did not show an increase in violence during a placebo period, nor did they have a history of violent behavior. The moderately high dosage of haloperidol ( $60 \mathrm{mg} /$ day) given in this study far exceeds in potency the dosages of both low-potency agents. Because haloperidol appears to display a therapeutic window for plasma concentrations (Potkin et al., 1986), it could be argued that these patients may have had high plasma levels that exceeded the upper end of the therapeutic window. Unfortunately, haloperidol plasma levels were not drawn during the drug trial. The high dosage of haloperidol may have induced a form of behavioral toxicity, manifested by an increase in activity and excitement that could conceivably lead to an increase in violent behavior. This type of behavioral abnormality is not relieved by increasing the dosage of medication and may be related to high blood levels of the neuroleptic (Simpson, 1975). The present study was undertaken as part of a larger multicenter clozapine study, and preliminary results indicate that only $4 \%$ of haloperidol-treated patients improved. While these were treatment-resistant patients, the small improvement further suggests a lack of response to moderately highdose haloperidol, and one could speculate whether a lower dosage may have been associated with greater overall improvement.

Examination of the neurological rating scale and the scale item akathisia in particular suggested that there were more extrapyramidal symptoms in the violent group during the haloperidol trial. Because akathisia symptoms have been implicated in violent behavior in schizophrenic patients (Van Putten, 1975), it is possible that the high extrapyramidal effects of haloperidol may explain the increase in violence, but we must note that the results were only a trend and failed to reach significance. It is also possible that the rating scale may not reveal subtle inner restlessness, which may lead to sudden overt and violent behavior. Van Putten et al. (1980) argue that akathisia is related to the plasma concentration, and that at higher concentrations (which may be inferred from the moderately high doses given to our subjects) akathisia could aggravate the patients' clinical condition and predispose to more violent behavior. Although patients received benztropine with haloperidol, it has been well-observed clinically that akathisia often appears despite prophylactic antiparkinsonian medication and is often resistant to such treatment.

Low-potency drugs, such as chlorpromazine and clozapine, are highly sedative and thus might be able to suppress violent behavior in the same way that other sedatives (e.g., benzodiazepines) are useful in violent patients. However, we did not measure a reduction in violence as compared with the placebo period, so that 
a specific effect on violence does not appear to be present. It is possible that, in some patients, low-potency neuroleptics may increase violent behavior via central nervous systems disinhibition or their potential epileptogenic effects. Approximately an equal number of patients who become violent on haloperidol were randomly assigned to receive either chlorpromazine or clozapine during the low-potency treatment period. Although the number of patients is too small for statistical analysis, it appears that both low-potency drugs were equally able to suppress violence observed during the haloperidol trial. Both drugs, and clozapine in particular, have very low extrapyramidal effects (Pi and Simpson, 1983).

We found in a controlled study that some patients have a marked increase in violence when treated with moderately high-dose haloperidol. At this time, we do not know whether such behavior is specific to haloperidol, whether it may have resulted from the relatively higher dosage, or whether it may also occur with other high-potency neuroleptics. The reasons for the increased violence may be related to presumed exceeding of the therapeutic window with haloperidol with consequent decrease in antipsychotic effectiveness, or may result from subtle extrapyramidal effects of the drug, particularly akathisia (see also Bjorndal et al., 1980). Further controlled studies are therefore warranted to elucidate the mechanism of increased violence with haloperidol and to ascertain whether the effect is unique to haloperidol or is due to the moderately large dosage used. Further studies should attempt to randomize patients between equivalent dosages of both high and low-potency neuroleptics. Presently, however, our results indicate that violent behavior may be more frequent on moderately highdose haloperidol than on moderate doses of low-potency neuroleptics.

\section{References}

Bjorndal N, Bjerre M, Gerlach J, et al (1980) High dosage haloperidol therapy in chronic schizophrenic patients. A double-blind study of clinical response, side effects, serum haloperidol and serum prolactin. Psychopharmacology 67:17-35.

Csernansky JG, Maddock RJ, Hollister LE (1985) Pharmacologic treatment of aggression. Hosp Formul 20:1091-1104.

Dubin WR (1981) Evaluating and managing the violent patient. Ann Emerg Med 10:481-484.

Elie R, Langlois Y, Cooper SF, et al (1980) Comparison of SCH12679 and thioridazine in aggressive mental retardates. Can $J$ Psychiatry 25:484-491.

Felthous AR (1984) Preventing assaults on a psychiatric inpatient ward. Hosp Community Psychiatry 35:1223-1226.

Gunn J (1979) Drugs in the violence clinic. In M Sandler (Ed), Psychopharmacology of aggression. New York: Raven.

Guy W (1976) ECDEU Assessment manual for psychopharmacology (pp 534-537). U.S. Dept. of Health, Education \& Welfare.

Itil TM, Wadad A (1975) Treatment of human aggression with major tranquilizers, antidepressants, and newer psychotropic drugs. $J$ Nerv Ment Dis 160:83-99.

King DJ (1981) The psychiatrically violent patient. Br Med J 282:821.

Lion J, Snyder W, Merrill G (1981) Underreporting of assaults on staff in a state hospital. Hosp Community Psychiatry. 32:497498.

Mulvey EP, Lidz CW (1984) Clinical considerations in the prediction of dangerousness in mental patients. Clin Psychol Rev 4:379-401.

Overall J, Gorham D (1962) The Brief Psychiatric Rating Scale. Psychol Rep 10:799-812.

Pi EH, Simpson G (1983) Atypical neuroleptics: Clozapine and the benzamides. In WE Fann, RC Smith, JM Davis (Eds), Modern Problems in Pharmacopsychiatry (Vol 21, pp 80-86) Basel: Karger.

Potkin S, Hahn R, Sramek J (1986) Plasma neuroleptic concentrations: In search of a therapeutic window. Clin Neuropharmacol 9:256-258.

Simpson G, Angus L (1970) A rating scale for extrapyramidal effects. Acta Psychiatr Scand (Suppl) 212:10-19.

Simpson GM (1975) CNS effects of neuroleptic agents. Psychiatr Ann 5:347-351.

Van Putten T (1975) The many faces of akathisia. Compr Psychiatry $16: 43-47$.

Van Putten T, Morder SR, May PRA, et al (1980) Plasma level of haloperidol and clinical response. P'sychopharm Bull 21:69-72.

Yar-Khan S (1981) The psychiatrically violent patient. $\mathrm{Br}$ Med $J$ 282:1400-1401.

Yesavage JA (1983) Inpatient violence and the schizophrenic patient: Study of Brief Psychiatric Rating Scale scores and inpatient behavior. Acta Psychiatr Scand 67:353-357.

Yesavage JA (1984) Correlates of dangerous behavior by schizophrenies in hospital. J Psychiatr Res 18:225-231. 